\title{
XI. On the asymmetry of the illumination-curves in oblique diffraction
}

\section{Sisir Kumar Mitra M.Sc.}

To cite this article: Sisir Kumar Mitra M.Sc. (1918) XI. On the asymmetry of the illuminationcurves in oblique diffraction, Philosophical Magazine Series 6, 35:205, 112-119, DOI: 10.1080/14786440108635740

To link to this article: http://dx.doi.org/10.1080/14786440108635740

曲 Published online: 08 Apr 2009.

Submit your article to this journal $\lceil\pi$

Џ Article views: 6

Q View related articles $\asymp$ 
XI. On the Asymmetry of the Illumination-Curves in Oblique Diffraction. By Sistr Komar Mitra, M.Sc., Sir Rashbehary Ghosh Research Scholar in the University of Calcutta*.

\author{
[Plate V.] \\ Introduction.
}

T the Phil. Mag. for May 1911, C. V. Raman has given 1 the results of a photometric study of the unsymmetrical diffraction-bands due to an obliquely held rectangular reflecting surface previously observed by him $\dagger$. The measurements showed a very marked asymmetry in the distribution of intensity in the diffraction pattern, the theoretical explanation of which is discussed in the papers quoted. The following were the principal conclusions arrived at by Raman as the result of the quantitative experimental study of the case :-

(a) The illumination at the points of minimum intensity in the diffraction pattern is zero at all angles of incidence, and the positions of the minima are accurately given by the formula

$$
\delta= \pm \pi, \pm 2 \pi, \pm 3 \pi, \& \mathrm{c} \text {. }
$$

where $\delta=\frac{\pi a}{\lambda}(\sin i-\sin \theta), a$ being the width of the aperture, $\lambda$ the wave-length, and $i, \theta$ the angles of incidence and diffraction respectively; the fringes are wider on the side on which $\theta>i$ and their number is limited on that side, as $\theta$ cannot be greater than $\frac{\pi}{2}$.

(b) The formula of the usual type $\left(I=\sin ^{2} \delta / \delta^{2}\right)$ for the illumination in the pattern fails to represent the observed intensity-curves at oblique incidences except in regard to the position of the minima $(\delta= \pm \pi, \pm 2 \pi, \dot{\alpha}$. $)$. The intensities at corresponding points on either side of the central fringe for which the values of $\delta$ are numerically the same are not equal.

(c) The observed distribution of intensity was found to fit in with the theoretical formula, if the latter is multiplied by a factor proportional to the square of the cosine of the

* Communicated by Prof. C. V. Raman.

$\dagger$ C. V. Raman, M.A., "On the Unsymmetrical Diffraction Bands due to a Rectangular Aperture," Phil. Mag. Nov. 1906. See also Phil. Mag. Jan. 1909. 
obliquity, which, of course, is not the same at all points in the diffraction pattern. In other words, the ordinates of the illumination curve were found to be proportional to the expression $\cos ^{2} \theta \sin ^{2} \delta / \delta^{2}$.

The question arises whether these results, practically those indicated in (b) and (c) above, are peculiar to the case of a surface of rectangular form, or whether similar phenomena might be expected with other forms of surface as well. The cases which it seemed of particular interest to examine are tose in which the reflecting surface is not a single individual area but consists of two, three, or more parallel elements lying in the same plane. A satisfactory surface of this kind which can be used at very oblique incidences may be prepared by etching out deep grooves on the optically plane surface of a thick plate of glass with hydrofluoric acid, the edges of the reflecting strips left on the surface being subsequently ground so as to be sharp, straight, and parallel. I have prepared several such surfaces containing two and three equidistant reflecting strips respectively. By placing one of these on the table of a spectrometer, the diffraction pattern produced by reflexion at very oblique incidences may be readily observed through the telescope of the instrument. The present paper describes the results of the quantitative study of the phenomena thus obtained. Incidentally the opportunity has also been taken of testing the results obtained by Raman for the case of a single aperture using improved optical and photographic appliances. The experiments and determinations have throughout been made using monochromatic light. This was secured by illuminating the slit of the spectrometer with light of a definite wave-length isolated by a monochromator from sunlight or arc light.

\section{Unsymmetrical Interference-fringes due to two parallel apertures.}

Fig. I (Pl. V.) reproduces a photograph of the diffraction pattern due to a surface containing two reflecting elements each of width $0.48 \mathrm{~cm}$., and $3.60 \mathrm{~cm}$. apart. The direct image of the slit of the spectrometer also appears in the figures to the right of the diffraction-pattern. The photograph is reproduced from a dense negative taken to show the perfect blackness of the minima of illumination, and the progressive increase (from left to right) in the width of the interference-fringes of the light diffracted by the two reflecting elements. It was obtained by replacing the telescope

Phil. Mag. S. 6. Vol. 35. No. 205. Jan. 1918. 


\section{Mr. S. K. Mitra on the Asymmetry of}

of the spectrometer by a camera with a lens of long focus $(176 \mathrm{~cm}$.). Figs. II, III, and IV reproduce three photographs taken at three different angles of incidence, the reflecting strips in this case being $0.754 \mathrm{~cm}$. wide and $1.446 \mathrm{~cm}$. apart. In all the figures, the central fringe of the pattern is indicated by a small cross $x$. The asymmetry of the luminosity curve will be evident on comparing the brightness of the corresponding bands on either side of the central fringe; for instance, the second band on the right and the second band on the left in figs. II and III, or the first band on the right and the first band on the left in fig. IV.

The positions of the interference minima in the pattern are given by the formula of the usual type

$$
\delta= \pm \frac{\pi}{2}, \pm \frac{3 \pi}{2}, \quad \pm \frac{5 \pi}{2}, \text { dc. }
$$

where $\delta=\pi(a \pm b)(\sin i-\sin \theta) / \lambda, a$ being the width of each of the apertures, $b$ their distance apart, and $i, \theta, \lambda$ having their usual significance. To test whether the formula holds good at the oblique incidences used, the negatives were measured under a travelling microscope. In photograph I, the distances between the successive interference minima were determined to find whether the relations

$$
\sin \theta_{1}-\sin \theta_{2}=\sin \theta_{2}-\sin \theta_{3}=\sin \theta_{3}-\sin \theta_{4}, \& c \text {., }
$$

indicated by the formula were valid. The results are shown in Table I.

\section{Table I.}

$$
a=0.48 \mathrm{~cm} . \quad b=3.60 \mathrm{~cm} \text {. }
$$

\begin{tabular}{|c|c|c|c|}
\hline $\begin{array}{c}\text { inima on the } \\
\text { right of the } \\
\text { ent ra }\end{array}$ & $\begin{array}{c}\text { Observed value of } \\
\sin \theta_{n+1}-\sin \theta_{n} \\
\times \text { constant. }\end{array}$ & $\begin{array}{c}\text { Minima on the } \\
\text { loft of the } \\
\text { central fringe. }\end{array}$ & $\begin{array}{c}\text { Obserred value of } \\
\sin \theta_{n}-\sin \theta_{n+1} \\
\times \text { constant. }\end{array}$ \\
\hline$\theta_{5}-\sin \theta_{4}$ & 0.712 & $\sin \theta_{1}-\sin \theta_{2}$ & 0.705 \\
\hline $\sin \theta_{4}-\sin \theta_{3}$ & 0.703 & $\sin \theta_{2}-\sin \theta_{3}$ & 0.710 \\
\hline $\sin \theta_{3}-\sin \theta_{2}$ & 0.711 & $\sin \theta_{3}-\sin \theta_{4}$ & 0.711 \\
\hline $\sin \theta_{2}-\sin \theta_{1}$ & 0.711 & $\sin \theta_{4}-\sin \theta_{5}$ & 0.710 \\
\hline$\ldots . .$. & $\ldots . .$. & 0.713 \\
\hline
\end{tabular}


the Illumination Curves in Oblique Refraction.

For photographs II, III, and IV, the actual values of $\theta$ for the interference minima were calculated from the known constants $a, b, i, \lambda$ and compared with the observed values. These are shown in Tables II., III., and IV.

\section{TABLI II.}

$$
a=0.754 \mathrm{~cm} . \quad b=1.446 \mathrm{~cm} . \quad \lambda=\cdot 0000435 \mathrm{~cm} .
$$

\begin{tabular}{|c|c|c|}
\hline Interference Minima. & $\begin{array}{l}\text { Calculated diffraction } \\
\text { angle, } 90^{\circ}-\theta \\
\text { (in minutes). }\end{array}$ & $\begin{array}{l}\text { Observed diffraction } \\
\text { angle, } 90^{\circ}-\theta \\
\text { (in minutes). }\end{array}$ \\
\hline 2nd on the right. & $35^{\prime} \cdot 98$ & $35^{\prime} \cdot 85$ \\
\hline 1st ", ", & $41^{\prime} 97$ & $41^{\prime} \cdot 83$ \\
\hline 1st ," , left. & $47^{\prime} \cdot 21$ & $46^{\prime} \cdot 95$ \\
\hline 2nd $", "$, & $51^{\prime} \cdot 92$ & $52^{\prime} \cdot 05$ \\
\hline 5 th $, ", "$ & $64^{\prime} \cdot 02$ & $63^{\prime} \cdot 95$ \\
\hline
\end{tabular}

\section{TABLE III.}

$a=0 \cdot 754 \mathrm{~cm} . \quad b=1.446 \mathrm{~cm} . \quad \lambda=\cdot 0000435 \mathrm{~cm}$. $i=89^{\circ} 22^{\prime} \cdot 75$.

\begin{tabular}{|c|c|c|}
\hline Interference Minima. & $\begin{array}{c}\text { Calculated } \\
90^{\circ}-\theta .\end{array}$ & $\begin{array}{c}\text { Observed } \\
90^{\circ}-\theta .\end{array}$ \\
\hline 2nd on the right. & $26^{\prime} \cdot 15$ & $26^{\prime} \cdot 12$ \\
\hline 1st $", "$, & $33^{\prime} \cdot 88$ & $33^{\prime} \cdot 85$ \\
\hline 1st , , left. & $40^{\prime} \cdot 25$ & $40^{\prime} \cdot 09$ \\
\hline 2nd $, ", "$ & $45^{\prime} \cdot 69$ & $45^{\prime} \cdot 70$ \\
\hline 5th $, ", "$ & $58^{\prime} \cdot 90$ & $58^{\prime} \cdot 75$ \\
\hline
\end{tabular}


TABLE IV.

$$
a=\cdot 754 \mathrm{~cm} . \quad b=1 \cdot 446 \mathrm{~cm} . \quad \lambda=\cdot 0000435 \mathrm{~cm} .
$$

\begin{tabular}{|c|c|c|}
\hline Interference Minima. & $\begin{array}{c}\text { Calculated } \\
90^{\circ}-\theta .\end{array}$ & $\begin{array}{c}\text { Observed } \\
90^{\circ}-\theta .\end{array}$ \\
\hline 1st on the right. & $25^{\prime} \cdot 72$ & $25^{\prime} \cdot 62$ \\
\hline 1st on the left. & $33^{\prime} \cdot 75$ & $33^{\prime} \cdot 45$ \\
\hline 2nd , . , & $40^{\prime} \cdot 08$ & $39^{\prime} \cdot 94$ \\
\hline 5th , , , & $54^{\prime} \cdot 94$ & $54^{\prime} \cdot 96$ \\
\hline
\end{tabular}

The Asymmetry of the Illumination-Curves.

As remarked above, there is a very marked difference in the luminosity of the corresponding bands on either side of the central fringe of the pattern due to the reflecting surface of two elements. Similar effects are also noticeable when the reflecting surface consists of three elements. Figs. V and VI in the Plate reproduce two photographs obtained with a reflecting surface consisting of three elements. The difference between the intensities of the 2nd principal maximum on either side of the central one is very evident in the reproductions and might be made out even in respect of the secondary maxima on either side. This asymmetry demands an explanation. As is shown by the measurements given in Tables I. to IV., the positions of the minima of illumination are in good agreement with those calculated from the formula of the usual type, which are obtained on the assumption that each of the elements into which the reflecting surface may be divided diffracts light strictly in proportion to its area, and that the phase and intensity of the disturbance incident on the surface are the same as when the waves travel undisturbed. Further, the intensities at the points of minimum illumination are shown by observation and by the photographs to be zero, in agreement with the results indicated by these formulæ. On the other hand, the difference in the intensity at corresponding points of the pattern on either side of the central fringe remains unexplained according to such formulæ unless regarded as an obliquity effect. 
A series of comparisons of the intensities of corresponding bands on the two sides of the pattern has been made for the cases in which the reflecting surface consists of one, two, and three reflecting elements respectively, for various angles of incidence. For this purpose, I have used a rotatingsector photometer of the Abney type supplied by Messrs. Adam Hilger, in which the free disk, which can be adjusted by handle while in rotation, is smaller in radius than the fixed disk. The sectors when in rotation thus present two annuli of different intensities, the ratio of which can be adjusted at pleasure by moving the handle of the instrument. The disk of the photometer is placed at the focal plane of the observing telescope, so that the diffraction pattern can be seen through it with an eyepiece, the fringes on the brighter side being observed through the inner annulus of the disk, and those on the fainter side through the outer annulus. To enable the intensities at corresponding points on the two sides of the pattern to be compared, a screen with two vertical slits is interposed immediately in front of the photometric disk so as to cut off everything except the regions under observation, which are then adjusted to equality of brightness by moving the handle of the photometer. Several readings can be taken in succession and their a verage struck. The diffraction angles $\theta$ and $\theta^{\prime}$ of the two bands under comparison may then be measured under a micrometer eyepiece. Tables V., VI., and VII. show the observed ratios of the illumination and those calculated on the assumption that the formula for illumination includes a factor proportional to the square of the cosine of the obliquity. It is seen that the agreement is good except when the ratio is so large that it cannot be measured accurately, owing to the near approach of the fainter band towards the direct image of the slit.

\section{Table V.}

Single Reflecting Surface, width $0.90 \mathrm{~cm}$.

Ratio of intensity of the first band on the right and the first band on the left.

\begin{tabular}{|c|c|c|}
\hline Angle of Incidence. & $\begin{array}{c}\text { Observed ratio } \\
\text { of illumination. }\end{array}$ & $\begin{array}{c}\text { Calculated ratio } \\
\cos ^{2} \theta / \cos ^{2} \theta^{\prime} .\end{array}$ \\
\hline $88^{\circ} 42^{\prime}$ & $1 \cdot 80$ & $1 \cdot 78$ \\
$88^{\circ} 53^{\prime}$ & $2 \cdot 31$ & $2 \cdot 46$ \\
$88^{\circ} 56^{\prime}$ & $2 \cdot 81$ & $2 \cdot 89$ \\
$88^{\circ} 4^{\prime}$ & 4.09 & $4 \cdot 21$ \\
\hline
\end{tabular}




\section{TABLE VI.}

Reflexion grating of two elements. $a=0.754 \mathrm{~cm} . \quad b=1.446 \mathrm{~cm}$.

\begin{tabular}{|c|c|c|c|c|}
\hline Angle of Incidence. & \multicolumn{2}{|c|}{$\begin{array}{l}\text { Ratio of the 1st maxima } \\
\text { ov the right and left. } \\
\text { Observed. | Calculated. }\end{array}$} & $\begin{array}{l}\text { Ratio of the 2nd maxima } \\
\text { on the right and left. } \\
\text { Observed. | Oalculated. }\end{array}$ \\
\hline $89^{\circ} 6^{\prime}$ & $1 \cdot 43$ & $1 \cdot 40$ & $2 \cdot 09$ & $2 \cdot 01$ \\
$89^{\circ} 23$ & $2 \cdot 25$ & $2 \cdot 01$ & 3.91 & $4 \cdot 88$ \\
$89^{\circ} 28^{\prime}$ & $2 \cdot 37$ & $2 \cdot 44$ & $7 \cdot 50$ & $11 \cdot 06$ \\
\hline
\end{tabular}

TABLE VII.

Reflexion grating of three elements.

$$
a=0.440 \mathrm{~cm} . \quad b=0.741 \mathrm{~cm} \text {. }
$$

\begin{tabular}{|c|c|c|c|c|}
\hline Angle of Incidence. & \multicolumn{2}{|c|}{$\begin{array}{c}\text { Ratio of the 1st two } \\
\text { Principal Marima on either } \\
\text { side of the central one. } \\
\text { Observed. }\end{array}$} & \multicolumn{2}{|c|}{$\begin{array}{c}\text { Ratio of the 2nd two } \\
\text { Principal Maxima on either } \\
\text { side of the central one. } \\
\text { Observed. }\end{array}$} \\
\hline $88^{\circ} 49^{\prime}$ & 1.48 & 1.43 & 1.83 & $1 \cdot 70$ \\
$89^{\circ} 5^{\prime}$ & 1.63 & 1.71 & 2.90 & $\begin{array}{c}3 \cdot 15 \\
\text { more than } \\
89^{\circ} 27^{\prime}\end{array}$ \\
2.65 & 2.87 & $\times$ & 100 times. \\
\hline
\end{tabular}

\section{Summary and Conclusion.}

1. The unsymmetrical interference fringes of the light obliquely diffracted by two parallel reflecting surfaces in the same plane have been observed and photographed.

2. The illumination curve in the diffraction pattern (of the Fraunhofer class) due to an obliquely-held reflecting surface (which may consist of two or more separate parts in the same plane), is found to be markedly asymmetrical, corresponding points on either side of the central fringe being of very different intensities. As the positions of the points of minimum (i.e. zero) illumination are found to be in close agreement with those given by the formula of the usual type, the asymmetry of the illumination curve may be explained as due to the varying obliquity at different points in the diffraction pattern. Measurements of the ratio of the 
intensities at corresponding points have been made with reflecting surfaces of rectangular form or consisting of either two or three elements in the same plane for various angles of incidence; the results show that the expression for the illumination at any point of the diffraction pattern contains a factor proportional to the square of the cosine of the obliquity at such point.

The experiments and observations described in the note were carried out in the Palit Laboratory of Physics. The writer hopes to carry out further work on the subject of oblique diffraction by various forms of aperture, and particularly in regard to the positions of the points of maximum intensity in the pattern, which would no doubt differ from those given by the usual formulæ owing to the asymmetry of the illumination curves.

Calcutta,

8th June, 1917.

XII. On the Two-Dimensional Motion of Infinite Liquid produced by the Translation or Rotation of a Contained Solid. By J. G. Leathem, M.A., D.Sc., Fellow of St. John's College, Cambridge *

1. DERIODIC conformal transformations - that is, transformations by which doubly connected regions in the plane of a variable $z=x+i y$, externally unbounded and bounded internally by a closed polygon or curve, may be represented conformally and repeatedly upon successive semi-infinite strips of width $\lambda$ in the half-plane $\eta>0$ of a variable $\zeta=\xi+i \eta$-have been studied by the present writer in a previous paper $\dagger$. It has there been shown how the knowledge of such a transformation for any particular curve makes possible the specification of a field of circulatory liquid flow (with or without logarithmic singularities) round a fixed solid body bounded by the curve.

It is now proposed to show that such knowledge makes possible also the determination of the field of irrotational motion due to any translation or rotation of the same solid in surrounding infinite liquid.

* Communicated by the Author.

† J. G. Leathem, "On Periodic Conformal Curve-Factors and CornerFactors," Proc. Royal Irish Academy, vol. xxxỉi. Sec. A, August 1916. 


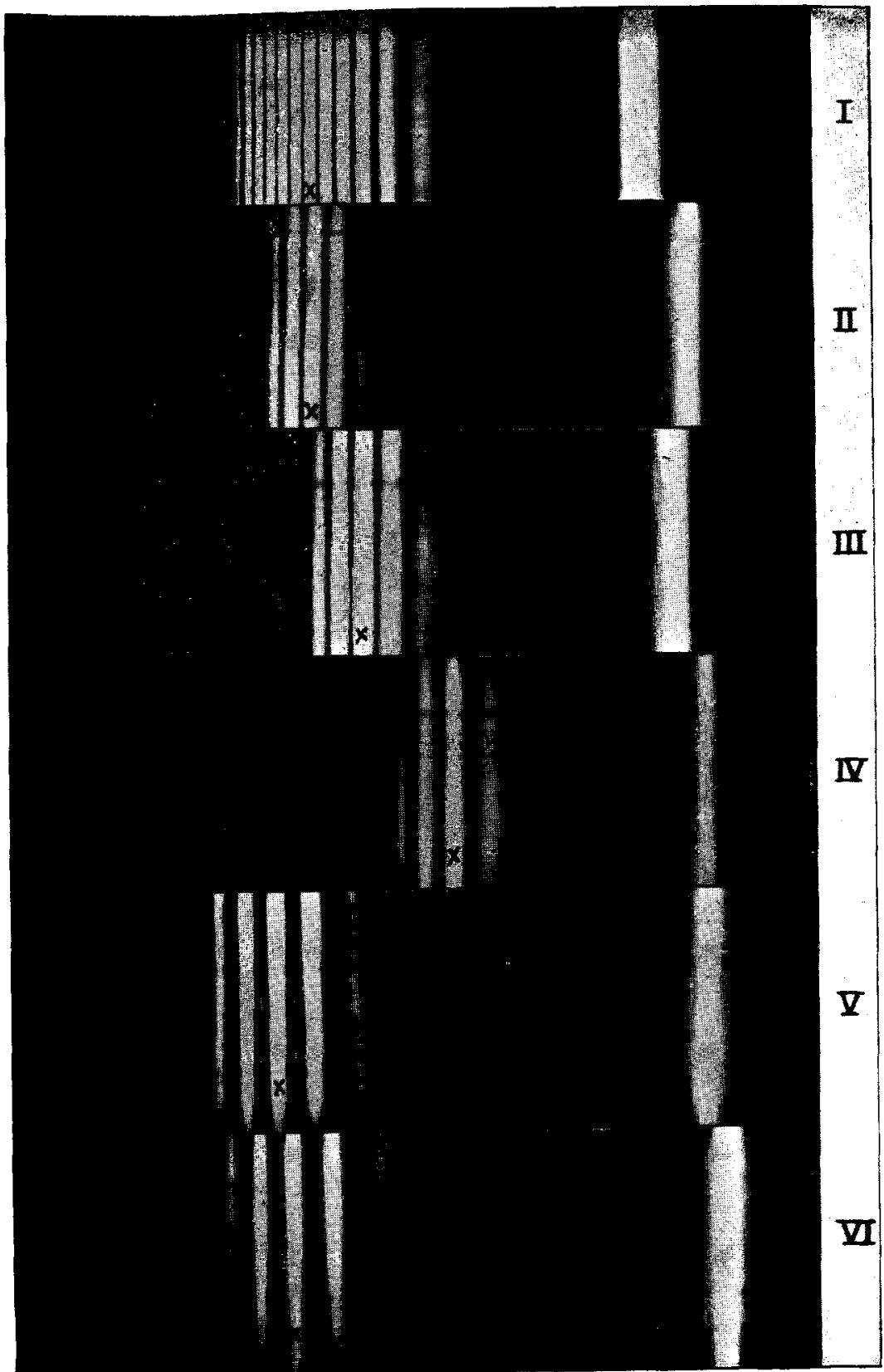

Illustrating the asymmetry of the fringes and of the illumination-curves in oblique diffraction by a reflecting surface consisting of two parts (Figs. I to IV) or three parts (Figs. V and VI) in the same plane. 